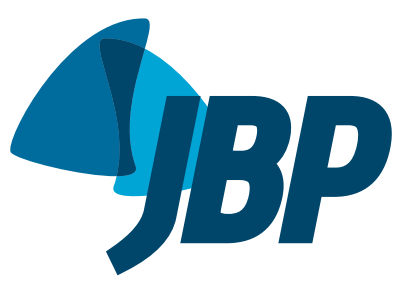

\title{
Gastric pseudo-obstruction as an initial manifestation of thymoma
}

\author{
Erlon de Ávila Carvalho',a, André Rossetti Portela1,b, \\ Marina Varela Braga de Oliveira ${ }^{1, c}$, Jéssica Rodrigues Girundi Guimarães ${ }^{1, d}$, \\ Shaline Braga Ramos ${ }^{1, e}$, Thamilys Benfica Pena ${ }^{1, f}$
}

\section{TO THE EDITOR:}

Thymomas and thymic carcinomas are rare, accounting for $20 \%$ of all mediastinal tumors. They are usually diagnosed as incidental findings on chest imaging performed for another reason or performed because of the presence of mass effect-related symptoms or paraneoplastic syndrome, such as myasthenia gravis. They have similar incidence in men and women and most commonly occur in the 40 - to 60 -year age group. There are no risk factors..$^{(1,2)}$

We would like to present the case of a 79-year-old female patient who presented to our facility with a two-month history of weight loss and constipation. She was admitted to the hospital with signs of intestinal obstruction, abdominal bloating, and vomiting. A nasogastric tube drained 3,000 $\mathrm{mL}$ of stool-like secretion. Abdominal X-ray and ultrasound revealed a dilated stomach with an air-fluid level (Figures $1 \mathrm{~A}$ and $1 \mathrm{~B}$ ). Upper gastrointestinal endoscopy revealed no points of mechanical obstruction; there was erosive reflux esophagitis, exuberant gastric dilatation associated with gastric stasis, and hypertrophic pyloric stenosis. Serology for Chagas disease was negative.
The assessment continued with abdominal $\mathrm{CT}$, which showed no evidence of any obstructive factor or tumor, and chest $\mathrm{CT}$, which identified a well-demarcated anterior mediastinal mass with a maximum diameter of approximately $5 \mathrm{~cm}$ and no invasion of adjacent structures (Figure 1C). Given the possibility of paraneoplastic syndrome, video-assisted thoracoscopic resection was performed, and pathological examination showed small cell neoplasm of uncertain malignant histogenesis and immunohistochemistry, confirming the diagnosis of type B1 thymoma.

Following tumor resection, the patient experienced a lower frequency of vomiting and was able to tolerate the diet. Gastrography was performed which showed contrast progression and a normal-sized stomach with peristalsis (Figure 1D). A second endoscopy revealed a normal stomach with strong peristaltic waves and no sign of previous hypertrophic pyloric stenosis. The patient was discharged on an oral diet. Serum antibody testing for myasthenia gravis and paraneoplastic syndrome was ordered.

Gastroparesis consists of delayed gastric emptying of solids in the absence of mechanical obstruction. The

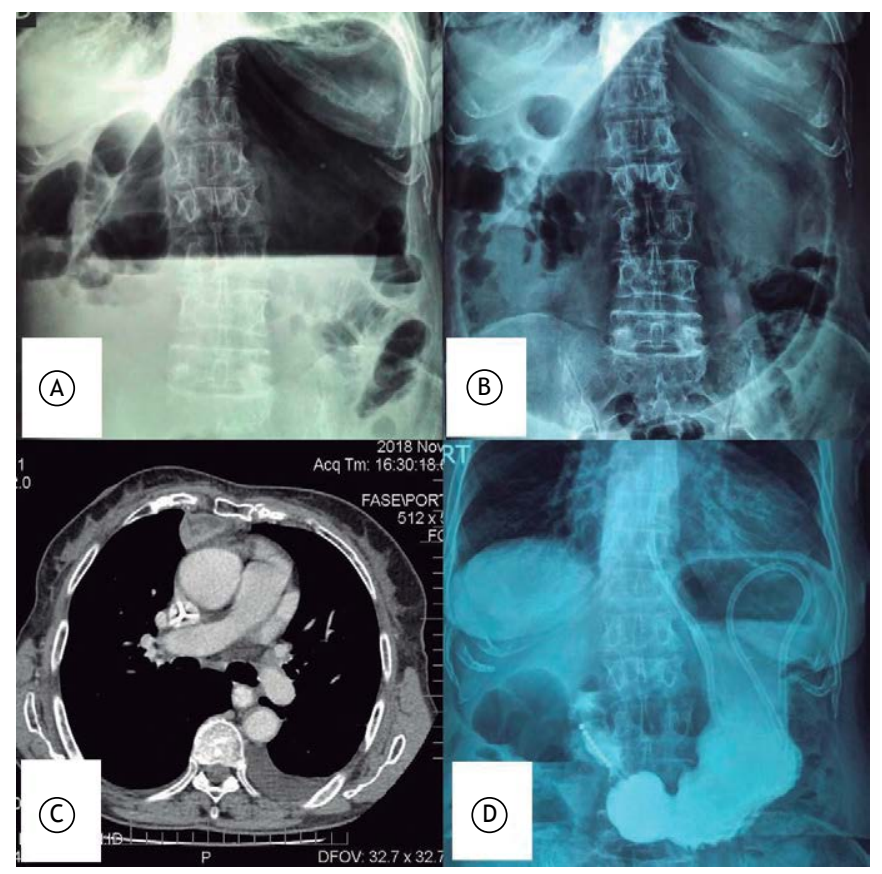

Figure 1. In A and $B$, abdominal $X$-ray showing gastric bloating. In $C, C T$ scan showing a well-demarcated anterior mediastinal tumor. In $D$, gastrography showing normal gastric emptying. 
incidence of gastroparesis is higher in female patients, and survival is significantly lower in this population than in the general population. The most common conditions associated with gastroparesis are functional dyspepsia, in $25 \%$; postoperative states (antrectomy, vagotomy, Roux-en-Y gastric bypass), in $25 \%$; other conditions (scleroderma, anorexia nervosa, uremia, paraneoplastic syndrome), in $15 \%$; and diabetes, in $25 \%$. . $^{(3)}$

In non-diabetic, non-Chagas disease patients with no mechanical obstruction and symptoms of gastroparesis, a diagnosis of paraneoplastic syndrome should be considered. Chief among the tumors to be tested are lung, ovarian, and breast carcinomas, as well as mediastinal tumors, such as neuroendocrine carcinomas and thymoma. ${ }^{(4)}$ Myasthenia gravis is the most common form of thymoma-related paraneoplastic neurological syndrome.

In the context of paraneoplastic syndromes causing gastrointestinal dysmotility, the pathogenesis can be explained by the autoimmune mechanism that affects the intrinsic enteric nervous system (interstitial cells of Cajal and myenteric plexus) and the extrinsic enteric nervous system (autonomic nervous system). ${ }^{(5)}$ Some antineuronal antibodies, such as $\mathrm{N}$-type calcium channel-binding antibodies, acetylcholine receptor antibody, type 1 antineuronal antibody, and anti-CV2 antibody, can be expressed by the tumor.

In patients who experience symptom improvement following tumor resection, the diagnosis can be made regardless of serum levels of those antibodies. In those patients in whom no tumor is found, determining serum levels is more important. The currently recommended treatment consists of resection of the primary tumor, when such a tumor is present, and the use of a selective acetylcholinesterase inhibitor for symptom relief. ${ }^{(5)}$ Preoperative biopsy is indicated in cases of lesions that appear to be malignant or that infiltrate adjacent structures.

Various paraneoplastic syndromes may occur in patients with thymic tumors; however, those syndromes are difficult to diagnose. It is paramount that they be clinically recognized and antibody levels be determined. Studies are needed so that the pathophysiology of those disorders can be understood.

\section{REFERENCES}

1. Shahrzad M, Le TS, Silva M, Bankier AA, Eisenberg RL. Anterio mediastinal masses. Am J Roentgenol. 2014;203(2):W128-38. https://doi.org/10.2214/AJR.13.11998

2. Safieddine N, Liu G, Cuningham K, Ming T, Hwang D, Brade A, et al. Prognostic factors for cure, recurrence and long-term survival after surgical resection of thymoma. J Thorac Oncol. 2014;9(7):1018-22. https://doi.org/10.1097/JTO.0000000000000215

3. Troncon LE. Gastroparesis: review of the aspects related to its concept, etiopathogeny and clinical handling [Article in Portuguese]
Rev Assoc Med Bras (1992). 1997;43(3):228-36. https://doi. org/10.1590/S0104-42301997000300011

4. Bohnenberger $H$, Dinter $H$, König A, Ströbel P. Neuroendocrine tumors of the thymus and mediastinum. J Thorac Dis. 2017;9(Suppl 15):S1448-S1457. https://doi.org/10.21037/jtd.2017.02.02

5. Pasha SF, Lunsford TN, Lennon VA. Autoimmune gastrointestina dysmotility treated successfully with pyridostigmine. Gastroenterology. 2006;131(5):1592-6. https://doi.org/10.1053/j. gastro.2006.06.018 